Z Herz-Thorax- Gefäßchir 2009 • 23:266

DOI 10.1007/s00398-009-0749-2

๑) Springer-Verlag 2009

\section{H.R. Zerkowski}

Riehen, Schweiz

\title{
Aus- und Weiterbildung schlecht? Nicht jammern - ändern!
}

Das kennt man ja schon und es soll aus der Sicht unserer Nachbarn auch typisch deutsch sein: Großartig in der Analyse, schwach in der Wertung - immer etwas selbstverliebt in der Skizzierung eines Fin de siècle, eines leicht depressiven bis pessimistischen Ausblicks, getragen von der Grundüberzeugung, dass eigentlich alles, aber auch wirklich alles viel, viel besser werden müsse. Vor allem: es müsse - von allein etwa?

Nun denn, passt doch - bis auf wenige Ausnahmen ein lahmer Wahlkampf, nur nichts beim Namen nennen; oder vor unserer eigenen Haustür: Die Schwierigkeiten des Fachgebiets betonen, das Ende mancher Schwerpunkte schon beinahe vor sich sehen, dabei das Faszinosum, das Potenzial, und damit unseren Nachwuchs, beinahe vergessen?

Bei uns tut sich seit einiger Zeit etwas. Geeigneter Nachwuchs ist ebenso wie Nachwuchsmangel nicht gottgegeben. Weiterbildungskommission, Akademie, besondere Kongressschwerpunkte für Studierende und Jungchirurginnen und -chirurgen, unsere Zeitschrift - die DGTHG kümmert sich, intensiver denn je. Kommissionen zeigen Selbstbewusstsein, betonen Erfolge auch im qualitativen Vergleich der Fachgebiete, informieren, fordern Mitarbeit ein. Der „wind of change“ blieb kein laues Lüftchen, das sich in der Hitze der BarcelonaSommer wieder legte.

So finden Sie in dieser Ausgabe hoffentlich erneut reichlich anregenden Lesestoff - von der OP-Technik (zu alten Problemen) bis zu Übersichten (mit futuristischen Lösungen), von „state of “ bis Denkanstoß sowie Wissenschaft und deren Hintergründen.

Auch Diskussion zu und Präsentation von Registern und das Bemühen um solche sind hochaktuell, fordert doch Neugebauer in der neuesten Ausgabe von EHEALTHCOM, gerade Qualitätsstandards zu Registern zu entwickeln. Denn „gute“ Register sind die notwendige Ergänzung $\mathrm{zu}$ „randomized controlled trials", um deren Ergebnissen ihren wahren Stellenwert in der realen Welt zu geben; eine Feststellung, die Beyersdorf, Grapow, Wattenwyl und Zerkowski aus der Mitte unserer Fachgesellschaft bereits im Jahr 2006 in einem Editorial für das Journal of Thoracic and Cardiovascular Surgery in gleicher Deutlichkeit trafen.

Dass derlei schon damals aus einer chirurgischen Disziplin kam, ist nachvollziehbar, gründet doch gute Chirurgie stets auf pragmatischen Lösungen im realen Umfeld definierter Probleme. Von den Ergebnissen in dieser "realen Welt“ ausgehend, sind nachhaltige Zukunftskonzepte zu entwickeln.

Den Boden für dieses Denken mit zu bereiten, dies zu unterstützen, ist und bleibt der Anspruch an Ihre Zeitschrift für Herz-, Thorax- und Gefäßchirurgie, dem wir uns vielleicht auch diesmal wieder ein Stück weit nähern. Viel Spaß beim Gewinnen neuer oder auch nur anderer Einsichten.

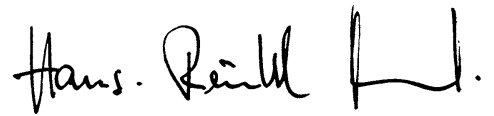

H.-R. Zerkowski

\section{Korrespondenzadresse}

Univ.-Prof. Dr. H.-R. Zerkowski

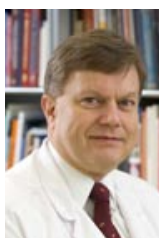

Steinkopff Verlag

Managing Editor Zeitschrift für Herz-, Thorax-, und Gefäßchirurgie Bahnhofstr. 25, CH-4125 Riehen, Schweiz zerkowski.htg@bluewin.ch 